\title{
Rotational laxity and collateral ligament laxity following total knee arthroplasty with rotating platform
}

\author{
Hermann O. Mayr • Maik Reinhold • Robert Hube • \\ Philipp von Roth • Anke Bernstein • Norbert Suedkamp • \\ Amelie Stoehr
}

Received: 6 January 2014 / Accepted: 18 February 2014 /Published online: 7 March 2014

(C) Springer-Verlag Berlin Heidelberg 2014

\begin{abstract}
Purpose The aim of this study was to evaluate laxity in knees with pre-operative (preop) valgus alignment compared to knees with pre-operative varus alignment after total knee arthroplasty (TKA).

Methods This was a retrospective study including 81 patients, with six years follow-up, for pre-operative valgus- or varus alignment of the leg. All patients had been supplied with the same cruciate retaining (CR) TKA with rotating platform. Clinical findings were assessed by KSS, OKS and IKDC 2000 score. Rotational knee laxity was evaluated by a validated instrument (Laxitester ${ }^{\circledR}$ ) with $2 \mathrm{Nm}$ torque in $30^{\circ}$ flexion. Collateral ligament laxity was tested manually in $30^{\circ}$ flexion with a bending moment of approximately $5 \mathrm{Nm}$. Biomechanical results were compared to the contralateral side.

Results Thirty-one patients had a preop valgus alignment of $8.96^{\circ}$ and 50 patients a varus leg axis of $4.99^{\circ}$ in the mean. In the preop valgus knees rotational analysis showed an increased laxity of $10.7^{\circ}$ compared to preop varus knees $(p=0.001)$. There was no significant difference in medial (valgus $2.6 \mathrm{~mm}$, varus $2.5 \mathrm{~mm}$ ) and lateral (valgus $2.8 \mathrm{~mm}$, varus $2.7 \mathrm{~mm}$ ) laxity. KSS and OKS showed no significant differences in the follow-up results. In the IKDC 2000 objective score $50 \%$ of the preop varus knees and $25.8 \%$ of the
\end{abstract}

\footnotetext{
H. O. Mayr $(\bowtie) \cdot$ A. Bernstein $\cdot$ N. Suedkamp

Department of Orthopaedic and Trauma Surgery, Albert Ludwig University of Freiburg, Hugstetter Str. 55, 79106 Freiburg, Germany e-mail: hermann.mayr@uniklinik-freiburg.de

M. Reinhold $\cdot$ R. Hube $\cdot$ A. Stoehr

OCM Clinic of Orthopaedic and Trauma Surgery, Munich, Germany

P. von Roth

Department of Traumatology and Reconstructive Surgery, Charité

Universitätsmedizin, Berlin, Germany
}

preop valgus knees were classified as nearly normal. The difference in the IKDC objective was highly significant $(p<0.001)$.

Conclusion Preop valgus knees show a significantly increased rotational laxity but no increased collateral ligament laxity compared to pre-operative varus knees six years after TKA with rotating platform. There is a significant difference in IKDC objective.

Keywords Knee laxity · Total knee replacement · Rotational laxity knee $\cdot$ Rotating platform TKA $\cdot$ Cruciate retaining TKA $\cdot$ Laxitester

\section{Introduction}

Total knee replacement is nowadays one of the most often performed procedures in orthopaedic surgery. In the presence of pre-operative valgus deformity compared with varus deformities functional difference was not observed using the KSS score [1]. Collateral ligament laxity has already been evaluated but so far there is no study published concerning rotational knee joint laxity after TKA [2]. The influence of pre- and postoperative leg axis concerning internal and external rotational laxity after TKA has not been investigated till now. Thus, the objective of this study was to evaluate the differences in rotational laxity and collateral ligament laxity of the operated knee depending on the pre-operative leg axis. In addition, the patient-related and objective outcomes were investigated.

The hypothesis of the trial was that pre-operative valgus knees show increased rotational laxity compared to pre-operative varus knees after TKA (CR) with rotating platform. 


\section{Material and methods}

In a retrospective study between February 2004 and September 2007, 81 consecutive patients (ASA I-III) between the ages of $71.8 \pm 5.4$ (females 72.5 years; males 71 years) underwent a total knee arthroplasty using the non-coupled LCS ${ }^{\circledR}$ DePuy system with a rotating platform. The implant used has a central rotating tibial inlay with high congruence between the inlay and the femoral component. Apart from rotation there are no further movement possibilities between tibial component and inlay. The posterior cruciate ligament was always retained, and the patella surface was not replaced by an artificial implant. Femoral and tibial components have been cemented in all patients. All operations were performed at the same hospital by the same surgeon. Forty-seven patients $(58 \%)$ received TKA in the right knee and 34 patients $(42 \%)$ on the left side. Exclusion criteria for participation in this study were the patient's age below 60 and above 80 years, pre-operative arthrofibrosis, status after knee joint fractures, osteotomies, previous knee ligament surgery, functional impairing gonarthritis of the contralateral knee, arthritis of other joints, other joint replacement of the lower extremity or rheumatoid disorders.

The instruments used to assess the clinical results were the Knee Society score (KSS) [3], Oxford knee score (OKS) [4] and International Knee Documentation Committee (IKDC) 2000 forms [5]. Conventional X-rays were obtained in three planes, i.e. posteroanterior with the patient standing on one leg, lateral view and a tangential view of the patella in $45^{\circ}$ flexion as well as a long leg standing AP view for leg axis determination. Collateral ligament stability was measured manually in $30^{\circ}$ knee flexion with a bending moment of approximately $5 \mathrm{Nm}$. Rotational laxity was examined using the Laxitester ${ }^{\circledR}$. All clinical examinations were compared to the contralateral side.

\section{Surgical technique}

The knee joint is opened via a so-called "mini-midvastus access". The LCS ${ }^{\circledR}$ rotating platform knee is implanted in a tibia first, flexion gap first technique. The technique is ligament adapted. In contract valgus knees a removal of osteophytes and a subperiosteal release of the iliotibial band at gerdiis tubercule is performed. If necessary a proximal "Pie Crusting" of the lateral collateral ligament and popliteus tendon is made. In contract varus knees after removal of osteophytes a distal subperiosteal release of the long medial collateral ligament is performed on the tibia, followed by a "Pie Crusting" of the medial capsule and ligament structures caudal and distal of the medial epicondyle. Attention is paid to balanced flexion and extension gaps. All ostephytes are removed. Band balancing is dependent on contracture and deformity and done from ventral to dorsal. In the case of an extension deficit an additional dorsal capsular release is performed. The axis alignment is done manually via so-called "alignment rod". Navigation was not used in patients in the present study. Joint replacement was done in a cemented technique using Refobacin-Palacos ${ }^{\circledR}$ bone cement. Patella replacement was not performed.

\section{Postoperative treatment}

All 81 patients included in the study underwent the same postoperative treatment protocol. On day 2, the first passive exercises with the CPM machine and mobilization of the patient on crutches started. Physiotherapy with open and closed kinetic chain exercises and gait training were performed avoiding pain. On the seventh to eighth postoperative days, patients were moved to a rehabilitation centre for three weeks. Depending on individual progress full weight bearing was allowed after three to six weeks postoperatively.

\section{Measurement of rotational knee laxity}

The Laxitester ${ }^{\circledR}$ (ORTEMA Sports Protection, Markgroeningen, Germany) is used for quantitative measurement of rotational laxity of the knee joint. The device was tested and validated on stable knee joints and knee joints with ligamentous damage [6,7]. As big interindividual differences exist reagarding rotational laxity, measurements are compared to the contralateral side. The Laxitester ${ }^{\circledR}$ is placed on the examination table and the patient is in supine position (Fig. 1). The leg is stored in the body axis. The femoral condyles are fixed by sliding side posts. Fixation of the femoral condyles prevents rotation of the thigh. The knee joint is mounted at an angle of $30^{\circ}$ of flexion on an adjustable support (pivot shift position). The measurement plate under the foot is movable in two planes and has adjustable cheeks for lateral fixation of the foot. Dorsiflexion of the ankle with a torque of $2 \mathrm{Nm}$ avoids rotational laxity of the ankle on the measurement plate. Three consecutive laxity measurements in internal and external rotation with a torque of $2 \mathrm{Nm}$ in the axis of the lower leg are then performed. The last measured value is documented in order to avoid major effects of viscoelasticity. It should be noted that the patient may not feel pain during the entire examination. When the internal and external rotational laxity of the TKA joint is measured, measurement on the opposite knee follows in the same way. Thus, differences in rotational laxity between knees with TKA and healthy knees can be determined.

\section{Statistics}

The primary endpoint is the rotational laxity of the knee compared to the contralateral side. It is assumed that patients from the population "preoperative valgus" achieve an 
Fig. 1 Laxitester ${ }^{\circledR}$. Rotational laxity (primary endpoint) of the knee was evaluated with a validated measuring device under $2 \mathrm{Nm}$ torque in internal and external rotation of the knee in $30^{\circ}$ flexion compared to the contralateral side

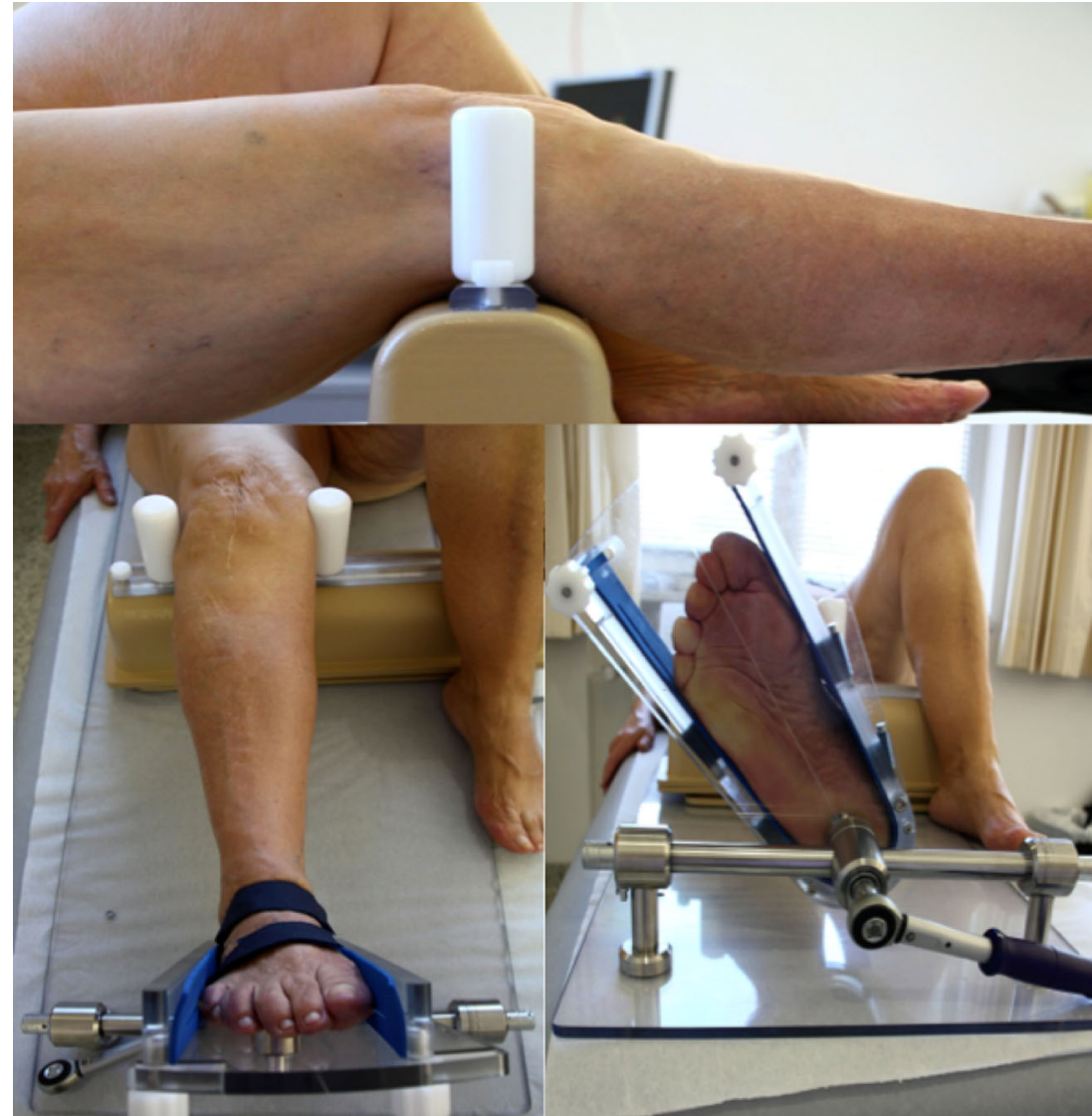

increased rotational laxity compared to the opposite knee with a mean off difference of $2.7^{\circ}$ and a standard deviation off difference of $7.1^{\circ}$. For a total of 80 patients and $\alpha=0.05$ a power of 0.95 can be obtained. For statistical evaluation of the program SSPS, version 19.0 was used. The following statistical tests were used: Kolmogorov-Smirnov to test for normal distribution; exact Wilcoxon test to compare the medians of two samples; $t$-test for independent samples to compare qualitative, unrelated traits in normal distribution; Mann-Whitney $U$-test for non-normally distributed samples, chi-square, Pearson, with statistical significance $p<0.05$, ANOVA and regression analysis, Spearman correlation coefficient for non-normally distributed samples.

The study was approved by the local ethics committee in a document dated November 16, 2010, admission number 346/10.

\section{Results}

Knee Society score

The results of the objective clinical examination, which are reflected in the knee score, reached a mean of $85.7 \pm 8.9$ points $(55-100)$ in pre-operative valgus knees and $88.8 \pm 12$ points in varus knees. The study population achieved mainly "good" and "very good" results. The subjective functional score reached a mean of $92.1 \pm 9.5$ points in valgus knees vs. 94.7 \pm 8.1 points in varus knees. Overall 45 patients $(55.6 \%)$ scored the maximum value of 100 points. Comparing preoperative varus and valgus aligned knees no significant differences were seen in the postoperative objective knee and functional score.

\section{Oxford knee score}

Evaluating the Oxford knee score, a mean of $44.8 \pm 3.7$ points in varus knees vs. $43.5 \pm 4.1$ points in valgus knees was achieved (min. 30, max. 48). Only one patient (1.2\%) indicated the minimum whereas 18 patients $(22.2 \%)$ scored the maximum value. It can be concluded that the majority of patients $(81.5 \%)$ is above the 42-point mark and therefore very satisfied in terms of range of motion, pain and daily activity after TKA. No significant differences were seen comparing the postoperative outcome with regard to varus or valgus pre-operative leg axis.

\section{IKDC 2000}

A significant difference $(p=0.032)$ was shown in the IKDC subjective score. Patients with valgus aligned knees reached 
$82 \pm 11$ points compared to $87 \pm 11$ points for patients with varus knees pre-operatively. As the overall difference is 5 points only, this seems to be marginally clinically relevant. Only one patient achieved the minimum score (1.2\%) of $48 \%$ and eight patients $(9.9 \%)$ the maximum of $98 \%$.

The results of the IKDC 2000 objective score show a significant difference $(p=0.015)$ comparing the mean of the pre-operative varus and valgus leg alignment group (Fig. 2). None of the patients reached group A, which stands for a knee with full functionality without symptoms and other findings. The majority of the patients with a pre-operative valgus alignment are scored group C (abnormal), whereas the majority of patients with a pre-operative varus axis can be found in group B (nearly normal).

A total of 74 patients (91.4\%) showed no effusion during clinical examination. The range of motion of the examined knee joints was determined by the neutral zero method in regard to extension and flexion deficits. Twenty-three patients $(28.4 \%)$ showed no flexion or extension deficit (E/F 10/0/135 ), whereas one patient $(1.2 \%)$ had a deficit in flexion and extension $\left(\mathrm{E} / \mathrm{F} 0 / 10 / 110^{\circ}\right)$. However, the majority of the patient population (50 patients, $61.7 \%$ ) suffered only a slight extension $\left(\mathrm{E} / \mathrm{F} 0 / 5 / 135^{\circ}\right)$ or flexion $\left(\mathrm{E} / \mathrm{F} 10 / 0 / 120^{\circ}\right)$ deficit.

\section{Radiological findings}

Pre-operatively 31 patients presented a valgus alignment with an axis deviation angle between 1 and $20^{\circ}$ (mean $8.96^{\circ} \pm$ $5.13^{\circ}$ ), and 50 patients a varus leg axis between 1 and $12^{\circ}$ (mean $4.9^{\circ} \pm 2.91^{\circ}$ ). Gender distribution represented no differences. Nearly all patients showed a corrected leg alignment after TKA (Fig. 3). The corrected postoperative hip knee ankle angle (HKA) showed a mean of $-0.65^{\circ} \pm 1.5^{\circ}$. Seven patients refused X-ray evaluation at the follow-up investigation. The detailed results of the radiological findings are presented in Table 1.

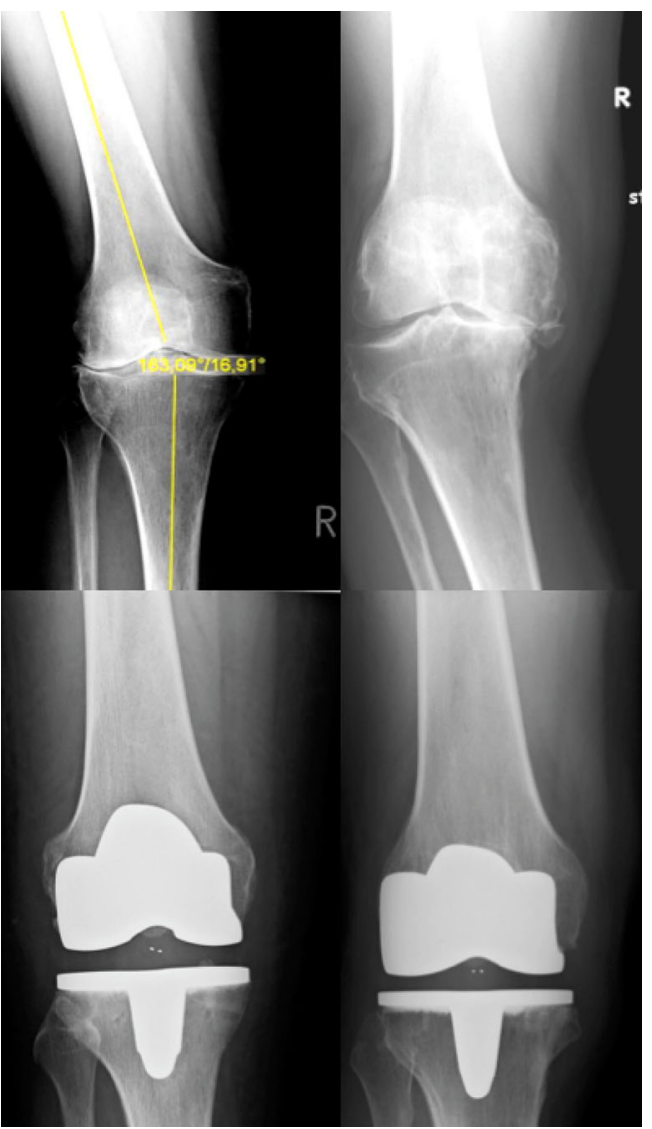

Fig. 3 X-rays. Left side Pre- and postoperative AP view of valgus gonarthritis and after leg axis correction with TKA. Right side Pre- and postoperative AP view of varus gonarthritis and after TKA

\section{Collateral ligament laxity}

No significant clinical and functional differences were found in the follow-up examination with regard to medial and lateral laxity of the knee in $30^{\circ}$ of flexion dependent on the preoperative leg alignment (Fig. 4).
Fig. 2 IKDC 2000 objective results. Varus knees scored most frequently nearly normal (b) in the postoperative examination whereas valgus knees were most frequently found abnormal (c) $(p<0.001)$

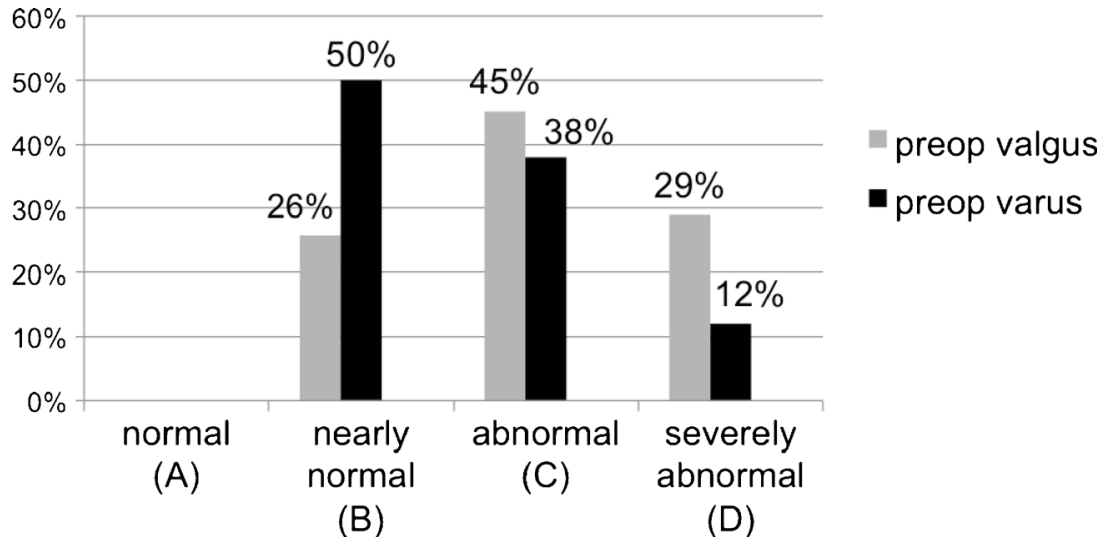


Table 1 Detailed presentation of radiological findings with regard to preop, postop and corrected HKA angles per patient

\begin{tabular}{|c|c|c|c|c|c|c|c|}
\hline & \multirow[b]{2}{*}{$\begin{array}{l}\text { Patient } \\
\text { number }\end{array}$} & \multirow[b]{2}{*}{$\begin{array}{l}\text { HKA preop } \\
\text { angle }\left({ }^{\circ}\right)\end{array}$} & \multirow[b]{2}{*}{$\begin{array}{l}\text { HKA correction } \\
\text { angle }\left({ }^{\circ}\right)\end{array}$} & \multirow[b]{2}{*}{$\begin{array}{l}\text { HKA postop } \\
\text { angle }\left(^{\circ}\right)\end{array}$} \\
\hline Patient & HKA preop & HKA correction & HKA postop & & & & \\
\hline & & & & \multirow{2}{*}{$\begin{array}{l}47 \\
48\end{array}$} & \multirow{2}{*}{$\begin{array}{r}-4.3 \\
12.4\end{array}$} & \multirow{2}{*}{$\begin{array}{r}4.3 \\
-14.4\end{array}$} & \multirow{2}{*}{$\begin{array}{r}0 \\
-2\end{array}$} \\
\hline \multicolumn{4}{|l|}{ Valgus } & & & & \\
\hline 01 & 10.2 & -12.2 & -2 & 49 & 2.5 & -3.5 & -1 \\
\hline 02 & -4.4 & 3.4 & -1 & 50 & 6.7 & -6.7 & 0 \\
\hline 03 & -5.6 & 6.1 & 0.5 & 51 & 2 & -4 & -2 \\
\hline 04 & 3.6 & & Refused & 52 & 5.4 & -5.4 & 0 \\
\hline 05 & -1 & -1 & -2 & 53 & 6.4 & -7.4 & -1 \\
\hline 06 & -5.8 & 3.8 & -2 & 54 & -0.5 & 3 & 2.5 \\
\hline 07 & -15.8 & 14.8 & -1 & 55 & 2.1 & -0.1 & 2 \\
\hline 08 & -12.6 & 10.6 & -2 & 56 & -16.3 & 19.3 & 3 \\
\hline 09 & 1 & -2 & -1 & 57 & -11.1 & 13.1 & 2 \\
\hline 10 & 6 & -7 & -1 & 58 & 4 & -2 & 2 \\
\hline 11 & 9 & -10.5 & -0.5 & 59 & 8 & -11 & -3 \\
\hline 12 & 5.5 & -5.5 & 0 & 60 & 4.4 & -1.3 & 3 \\
\hline 13 & -3.5 & 3.5 & 0 & 61 & 1 & -1 & 0 \\
\hline 14 & 11 & & Refused & 62 & 4.5 & -3.5 & 1 \\
\hline 15 & 5 & -6.5 & -1.5 & 63 & 6.7 & -6.7 & 0 \\
\hline 16 & -7.8 & 6.8 & -1 & 64 & 8.2 & -10.2 & -2 \\
\hline 17 & 6 & -6 & 0 & 65 & 7.4 & -5.4 & 2 \\
\hline 18 & 2.5 & -4.5 & -2 & 66 & 5 & -8 & -3 \\
\hline 19 & -10 & 7 & -3 & 67 & 1.5 & -2.5 & -1 \\
\hline 20 & 2 & -3 & -1 & 68 & 3.6 & 1.6 & 2 \\
\hline 21 & 4 & -4 & 0 & 69 & 10.3 & -9.3 & 1 \\
\hline 22 & -2.1 & -0.9 & -3 & 70 & -14.6 & 11.6 & -3 \\
\hline 23 & 5.1 & -7.1 & -2 & 71 & -1.4 & 2.4 & 1 \\
\hline 24 & 2.7 & -4.2 & -1.5 & 72 & -19.8 & 17.8 & -2 \\
\hline 25 & 5.7 & -7.2 & -1.5 & 73 & -8.5 & 6.5 & -2 \\
\hline 26 & 8.3 & & Refused & 74 & 2 & -4 & -2 \\
\hline 27 & -5.5 & 6.5 & 1 & 75 & -4.8 & 4.5 & -0.5 \\
\hline 28 & -10.8 & & Refused & 76 & 2.1 & -2.1 & 0 \\
\hline 29 & -12.5 & 10.5 & -2 & 77 & -8.6 & 8.6 & 0 \\
\hline 30 & -11.1 & 12.1 & 1 & 78 & -17.6 & 15.6 & -2 \\
\hline 31 & 0.5 & -1 & -0.5 & 79 & -9 & & Refused \\
\hline 32 & 6 & -7 & -1 & 80 & 1.6 & & Refused \\
\hline 33 & 0.6 & -2.1 & -1.5 & 81 & -7.3 & 6.3 & -1 \\
\hline 34 & 8.6 & -10.6 & -2 & & & & \\
\hline 35 & -7.4 & 5 & -2 & & & & \\
\hline 36 & 2 & -4 & -2 & & & & \\
\hline 37 & 5 & -5 & 0 & Rotatio & knee laxity & & \\
\hline 38 & -13.3 & 13.3 & 0 & & & & \\
\hline 39 & 7.4 & -6.4 & 1 & $\begin{array}{l}\text { The me } \\
\text { operate }\end{array}$ & $\begin{array}{l}\text { rements of } \\
\text { ee were per }\end{array}$ & nal and interna & ation of the \\
\hline 40 & 7.3 & -8.8 & -1.5 & side. $\mathrm{Tl}$ & ifference is $h$ & y significant $(p<$ & 1) (Fig. 5). \\
\hline 41 & 5.6 & -7.6 & -2 & The & l difference & rotation angles & eop valgus/ \\
\hline $\begin{array}{l}42 \\
43\end{array}$ & $\begin{array}{l}2 \\
4.4\end{array}$ & $\begin{array}{l}-2 \\
-6.4\end{array}$ & $\begin{array}{r}0 \\
-2\end{array}$ & varus $k$ & $s$ after TKA & s $7.9^{\circ} \pm 6.7^{\circ}$ in & nal rotation \\
\hline 44 & 4.5 & -4.5 & 0 & and -2 . & $2.3^{\circ}$ in inter & tation compare & he contralat- \\
\hline 45 & -9.5 & & Refused & eral kne & $=0.007) . \mathrm{B}$ & In the two pre-c & ive leg axis \\
\hline 46 & -15.1 & 15.1 & 0 & $\begin{array}{l}\text { deviatic } \\
\pm 4.5^{\circ} \mathrm{e}\end{array}$ & $\begin{array}{l}\text { a highly sign } \\
\text { s after TKA }\end{array}$ & $\begin{array}{l}\text { ne summative di } \\
0.007) \text {. Regress }\end{array}$ & nalysis only \\
\hline
\end{tabular}

Table 1 (continued)

Rotational knee laxity

The measurements of external and internal rotation of the operated knee were performed compared to the contralateral . The difference is highly significant $(p<0.001)$ (Fig. 5).

The total difference of rotation angles in preop valgus/ varus knees after TKA was $7.9^{\circ} \pm 6.7^{\circ}$ in external rotation and $-2.8^{\circ} \pm 2.3^{\circ}$ in internal rotation compared to the contralatdeviations, a highly significant summative difference of $10.7^{\circ}$ $4.5^{\circ}$ exists after TKA $(p=0.007)$. Regression analysis only 


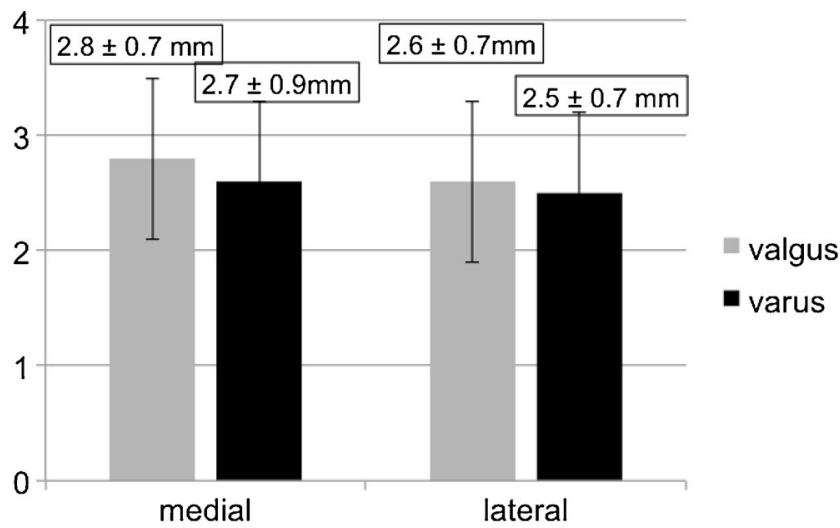

Fig. 4 Collateral ligament laxity. Medial and lateral laxity was measured in $30^{\circ}$ knee flexion and showed no significant difference between preoperative valgus and varus aligned knees

shows weak correlation between the expression of preoperative leg axis and rotation angles $(R=0.27)$.

\section{Discussion}

The objective of the present study was to evaluate the differences in rotational laxity and collateral ligament laxity after TKA with rotating platform in correlation to the pre-operative leg axis. In addition the patient related and objective outcomes were of interest. Between 2000 and 2008 the LCS $^{\circledR}$ rotating platform knee was used from the study group for all primary total knee replacements. In simulations of the loading pressure on polyethylene inlays a significant pressure reduction in using highly conformal mobile inlays compared to "fixed bearing" systems could be achieved [8,9]. Laboratory studies have shown that significantly less abrasion of the polyethylene could be detected in rotating platform inlays [10]. But rotating platform prostheses show no superior results when compared to fixed bearing prostheses in the Hospital for Special Surgery (HSS) scores [11].

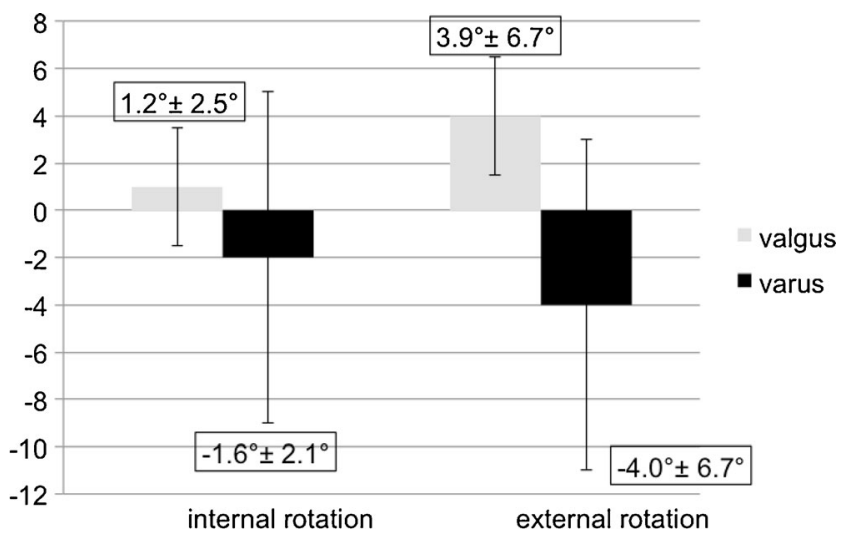

Fig. 5 Rotational laxity. Preoperative valgus knees showed significantly more rotational laxity with $10.7^{\circ}(p<0.001)$ compared to the preop varus knees
Thus the question of the study group was if the preoperative axis has clinically relevant influence on rotational laxity using this design.

Koninckx et al. [12] found that the far medial subvastus approach is an excellent approach to perform Krackow type I and II TKA with primary PS implants. Sekiya et al. [13] compared the medial and lateral approach in valgus knees from $6^{\circ}$ to $24^{\circ}$ in 48 knees. They found less invasiveness to the quadriceps muscle when using the lateral approach but no significant differences in alignment, surgical time and lateral laxity. Due to clinical routine all cases of the present study were performed with a mini-midvastus approach.

In the Oxford knee score the study patients reached a value of $44 \pm 3.9$ points on average. Given the maximum possible 48 points, these results are certainly to be interpreted as good to very good. A prospective study by Xie et al. [14] supports these findings but has only a short follow-up of two years.

The present results for the objective Knee Society score reached a mean of $87.1 \pm 10.4$ points and $93.3 \pm 8.9$ points in the subjective functional score. Xie showed comparable values with 89.1 points in the knee score after two years but lower results with 67.3 points in the functional score [14]. A reason could be that the Chinese population assesses the patient-related outcome differently.

Harwin et al. [15] studied 287 patients five years after knee replacement with a single-radius design and scored similar results to our findings despite using a different prosthesis (KSS knee score 93 points; KSS functional score 85 points).

After evaluation of the OKS and KSS no significant differences between pre-operative varus and valgus aligned knees could be established. The only significant differences were seen in the IKDC 2000. In the IKDC 2000 subjective a difference of 5 points $(p=0.032)$ was seen between the two groups, which is marginally clinically relevant. In the IKDC 2000 objective the majority of valgus patients $(56 \%)$ are in the poorer group $\mathrm{C}$, whereas the majority of varus patients can be found $(70 \%)$ in group B $(p<0.001)$.

In the conventional arthroplasty scores as OKS and KSS, however, apart from the IKDC 2000, no significant difference was seen. With regard to the OKS and KSS scores, as the most commonly used for follow-up examinations after TKA, the question has to be raised whether these scores are sensitive enough to meet today's patient expectations. It should be noted that the KSS was revised and published in 2012, but has not been used for the present study [16].

Due to criteria of good leg axis after TKA all our patients were within the range of $\pm 3^{\circ}[17]$.

Sufficient band balancing seems to be one of the most important criteria for a good clinical outcome after TKA. If the collateral ligaments are too lax, the risk of instability and increased abrasion is given. In case of very rigid soft tissue the patient often suffers from subjective limitations in range of motion with a simultaneous increase in pain intensity. Kuster 
et al. [18] examined a total of 22 patients to which both sides were supplied with a total knee replacement. He examined the ligamentous laxity with side-to-side difference, and found that the laxer knee joint is tolerated more than the tighter contralateral side. Ghosh et al. [19] conducted computer-navigated stability measurements on amputated lower extremities from deceased patients. The knee joint was thereby gradually destabilized by medical measures as arthrotomy, ACL resection, prosthesis implantation, PCL resection and resection of the popliteal muscle. Valgus and varus stress as well as internal and external rotation were then tested in different degrees of knee flexion. It was shown that after total knee arthroplasty, ligament laxity was significantly higher during valgus and varus stress.

An important role in knee joint stability is played by the PCL. Matsuda et al. [20] compared 24 patients with posterior cruciate retaining TKA and 24 patients after cruciate posterior cruciate resection in a randomized prospective work. Results showed significantly less varus/valgus laxity in patients with cruciate retaining surgery $(p=0.004)$. Takeda's study from 2011 turned out that patients have significantly more soft tissue laxity after cruciate resection. However, a significant difference in the clinical outcome could not be detected [21]. All of our 81 patients received a cruciate retaining implantation technique, so that the posterior cruciate-dependent knee stability was guaranteed.

However, the main target of the present study was to determine differences in collateral ligament and rotational laxity of the knee joints in relation to the pre-operative leg axis. Thirty-one patients had a pre-operative valgus alignment and 50 patients a varus leg axis. According to the reported results it can be stated that pre-operative valgus knees have no significant collateral ligament laxity after TKA. These results are confirmed by the findings of Heesterbeek et al. [2].

Rotational laxity with respect to the pre-operative leg axis was measured with the Laxitester ${ }^{\circledR}$ (ORTEMA Sports Protection, Markgroeningen, Germany). All examinations of external and internal rotation were performed in comparison with the contralateral side. The Laxitester ${ }^{\circledR}$ for quantitative measurement evaluation of rotational laxity of the knee joint has already been used in patients with ruptures of the anterior cruciate ligament and is clinically validated [6, 7]. In the present measurements, the pre-operative valgus aligned knees achieved an average difference of external rotation of $3.9^{\circ} \pm$ $6.7^{\circ}$, and internal rotation of $1.2^{\circ} \pm 2.5^{\circ}$ compared to the healthy contralateral knee. Pre-operative varus knees achieved an average difference in rotation of external rotation of $-4^{\circ} \pm$ $6.7^{\circ}$, and internal rotation of $-1.6^{\circ} \pm 2.1^{\circ}$. This results in an increased mean external rotation of $7.9^{\circ}$, and increased mean internal rotation of $2.8^{\circ}(p<0.001)$ for pre-operative valgus compared to varus knees. Between the two pre-operative axis deviations, a highly significant summative difference of $10.7^{\circ}$ $\pm 4.5^{\circ}$ exists after TKA $(p=0.007)$. The large standard deviation in the differentiated determination of the rotation angles in valgus knees may be especially dependent on the different amount of release in relation to the pre-operative valgus type. Summative however, the laxity angle difference between pre-operative valgus and varus knees is more than twice the standard deviation.

The reduced postoperative knee laxity in pre-operative varus knees seems to be due to postoperative fibrosis of periarticular soft tissue. Ligamento-capsular structures are much more complex on the lateral side of the knee compared to the medial side (e.g. iliotibial band, anterolateral ligament, lateral collateral ligament, popliteo-fibular ligament, popliteus complex and arcuatum ligament). Depending on the valgus deformity a release of these structures is necessary for ligament balancing. This could be the reason for increased rotational laxity.

Fixed bearing designs exhibit greater torque and higher cortical strain than rotating platform designs [22]. If there is an influence using the rotating platform on rotational laxity, it has not been studied so far [23]. So comparable results could not be found after extensive literature research because of the innovative approach. Lorbach et al. [24] are working on new instruments for rotation measurement. In their study, a total of 30 healthy patients were analysed without preliminary operations on both knees. The experimental setup is similar to the one applied in this study, but measurements with $5 \mathrm{Nm}$, $10 \mathrm{Nm}$, and $15 \mathrm{Nm}$ were performed. Due to these values and a significantly younger (mean age 24 years) and healthy study population, rotation values are not comparable to the present findings.

Weaknesses of the trial are such that the mini-midvastus approach was used for all study cases; the lateral approach was not performed in valgus deformities. This could result in a negative effect on laxity and function in valgus knees. The present study findings were not compared to fixed bearing design. Therefore it cannot be excluded that the measured rotational laxity is mainly dependent on the rotating platform design. Standard deviation in rotational laxity is very high, since the individual degree of release has not been documented.

\section{Conclusion}

Six years after TKA with rotating platform pre-operative valgus knees show a significantly increased rotational laxity but no increased collateral ligament laxity compared to preoperative varus knees. There is a significant difference in IKDC 2000. Therefore it should be the subject of future studies to compare rotational laxity in fixed bearing designs versus rotating platform. In addition it has to be rotational verified if the lateral approach reduces postoperative laxity in valgus knees. 


\section{References}

1. Dutka J, Sosin P, Ciszewski A, Sorysz T (2006) Impact of preoperative knee deformity on the outcome of total knee replacement using the PFC system. Ortop Traumatol Rehabil 8(2):201-209

2. Heesterbeek PJ, Keijsers NL, Wymenga AB (2010) Ligament releases do not lead to increased postoperative varus-valgus laxity in flexion and extension: a prospective clinical study in 49 TKR patients. Knee Surg Sports Traumatol Arthrosc 18(2):187-193

3. Insall JN, Dorr LD, Scott RD, Scott WN (1989) Rationale of the Knee Society clinical rating system. Clin Orthop Relat Res 248:13-14

4. Naal FD, Impellizzeri FM, Sieverding M, Loibl M, von Knoch F, Mannion AF, Leunig M, Munzinger U (2009) The 12-item Oxford Knee Score: cross-cultural adaptation into German and assessment of its psychometric properties in patients with osteoarthritis of the knee. Osteoarthr Cartil 17(1):49-52

5. Irrgang JJ, Anderson AF, Boland AL (2001) Development and validation of the international knee documentation committee subjective knee form. Am J Sports Med 29(5):600-613

6. Branch TP, Mayr HO, Browne JE, Campbell JC, Stoehr A, Jacobs CA (2010) Instrumented examination of anterior cruciate ligament injuries: minimizing flaws of the manual clinical examination. Arthroscopy 26(7):997-1004

7. Mayr HO, Hoell A, Bernstein A, Hube R, Zeiler C, Kalteis T, Suedkamp NP, Stoehr A (2011) Validation of a measurement device for instrumented quantification of anterior translation and rotational assessment of the knee. Arthroscopy 27(8):1096-1104

8. Stukenborg-Colsman C, Ostermeier S, Hurschler C, Wirth CJ (2002) Tibiofemoral contact stress after total knee arthroplasty: comparison of fixed and mobile-bearing inlay designs. Acta Orthop Scand 73(6): 638-646

9. Morra EA, Postak PD, Plaxton NA, Greenwald AS (2003) The effects of external torque on polyethylene tibial insert damage patterns. Clin Orthop Relat Res 410:90-100

10. Atwood SA, Currier JH, Mayor MB, Collier JP, Van Citters DW, Kennedy FE (2008) Clinical wear measurement on low contact stress rotating platform knee bearings. J Arthroplasty 23(3):431-440

11. Chen LB, Tan Y, Al-Aidaros M, Wang H, Wang X, Cai SH (2013) Comparison of functional performance after total knee arthroplasty using rotating platform and fixed-bearing prostheses with or without patellar resurfacing. Orthop Surg 5(2):112-117

12. Koninckx A, Schwab PE, Deltour A, Thienpont E (2013) The minimally invasive far medial subvastus approach for total knee arthroplasty in valgus knees. Knee Surg Sports Traumatol Arthrosc. Oct 9 [Epub ahead of print]

13. Sekiya H, Takatoku K, Takada H, Sugimoto N, Hoshino Y (2014) Lateral approach is advantageous in total knee arthroplasty for valgus deformed knee. Eur J Orthop Surg Traumatol 24(1):111115

14. Xie F, Lo NN, Pullenayegum EM, Tarride JE, O'Reilly DJ, Goeree R, Lee HP (2010) Evaluation of health outcomes in osteoarthritis patients after total knee replacement: a two-year follow-up. Health Qual Life Outcomes 8:87

15. Harwin SF, Issa K, Given K, Hitt KD, Greene KA, Pivec R, Kester M, Mont MA (2013) Clinical and patient-reported outcomes of primary TKA with a single-radius design. Orthopedics 36(7):e877e882

16. Scuderi GR, Bourne RB, Noble PC, Benjamin JB, Lonner JH, Scott WN (2012) The new Knee Society Knee Scoring System. Clin Orthop Relat Res 470(1):3-19

17. Daniilidis K, Tibesku CO (2013) Frontal plane alignment after total knee arthroplasty using patient-specific instruments. Int Orthop 37(1):45-50

18. Kuster MS, Bitschnau B, Votruba T (2006) Influence of collateral ligament laxity on patient satisfaction after total knee arthroplasty: a comparative bilateral study. Arch Orthop Trauma Surg 124(6):415417

19. Ghosh KM, Blain AP, Longstaff L, Rushton S, Amis AA, Deehan DJ (2013) Can we define envelope of laxity during navigated knee arthroplasty? Knee Surg Sports Traumatol Arthrosc. Jul 6 [Epub ahead of print]

20. Matsuda Y, Ishii Y, Noguchi H, Ishii R (2005) Effect of flexion angle on coronal laxity in patients with mobile-bearing total knee arthroplasty prostheses. J Orthop Sci 10(1):37-41

21. Takeda M, Ishii Y, Noguchi H, Matsuda Y, Sato J (2012) Changes in varus-valgus laxity after total knee arthroplasty over time. Knee Surg Sports Traumatol Arthrosc 20(10):1988-1993

22. Malinzak RA, Small SR, Rogge RD, Archer DB, Oja JW, Berend ME, Ritter MA (2014) The effect of rotating platform TKA on strain distribution and torque transmission on the proximal tibia. J Arthroplasty 29(3):541-547

23. Huang ZM, Ouyang GL, Xiao LB (2011) Rotating-platform knee arthroplasty: a review and update. Orthop Surg 3(4):224 228

24. Lorbach O, Wilmes P, Theisen D, Brockmeyer M, Maas S, Kohn D, Seil R (2009) Reliability testing of a new device to measure tibial rotation. Knee Surg Sports Traumatol Arthrosc 17(8):920-926 